\title{
Evaluation of the effectiveness and acceptability of intramuscular clozapine injection: illustrative case series
}

\author{
Rebecca Henry, ${ }^{1}$ (1) Ruth Massey, ${ }^{2}$ Kathy Morgan, ${ }^{3}$ Johanne Deeks, ${ }^{4}$ Hannah Macfarlane, ${ }^{4,5}$ \\ Nikki Holmes, ${ }^{6}$ (1) Edward Silva ${ }^{2}$ (1)
}

BJPsych Bulletin (2020) 44, 239-243, doi:10.1192/bjb.2020.6

\author{
${ }^{1}$ Southern Health NHS Foundation Trust, \\ UK; ${ }^{2}$ Mersey Care NHS Foundation \\ Trust, UK; ${ }^{3}$ Pennine Care NHS \\ Foundation Trust, UK; ${ }^{4}$ Birmingham and \\ Solihull Mental Health NHS Foundation \\ Trust, UK; ${ }^{5}$ Aston University, UK \\ ${ }^{6}$ Nottinghamshire Healthcare NHS \\ Foundation Trust, UK \\ Correspondence to Rebecca Henry \\ (rebecca.henry@southernhealth.nhs.uk) \\ First received 15 Nov 2019, final revision \\ 16 Dec 2019, accepted 17 Jan 2020 \\ (c) The Authors 2020. This is an Open \\ Access article, distributed under the \\ terms of the Creative Commons \\ Attribution licence (http:// \\ creativecommons.org/licenses/by/4.0/), \\ which permits unrestricted re-use, \\ distribution, and reproduction in any \\ medium, provided the original work is \\ properly cited.
}

Aims and method A series of eleven patients prescribed intramuscular clozapine at five UK sites is presented. Using routinely collected clinical data, we describe the use, efficacy and safety of this treatment modality.

Results We administered 188 doses of intramuscular clozapine to eight patients. The remaining three patients accepted oral medication. With the exception of minor injection site pain and nodules, side-effects were as expected with oral clozapine, and there were no serious untoward events. Nine patients were successfully established on oral clozapine with significant improvement in their clinical presentations.

Clinical implications Although a novel formulation in the UK, we have shown that intramuscular clozapine can be used safely and effectively when the oral route is initially refused.

\section{Declaration of interest None.}

Keywords Clozapine; intramuscular; schizophrenia; treatment refusal.
Clozapine remains the gold-standard intervention for treatment-resistant schizophrenia (TRS), offering a wide range of benefits. ${ }^{1-5}$ Case reports and series describing the use of intramuscular clozapine for patients unable or unwilling to take oral treatment have been published from authors based in Israel, the Netherlands and Australia. ${ }^{6-9}$ Although it is possible to successfully administer clozapine via a nasogastric tube as an alternative to the oral route, the more conventional option of an injection is usually preferable if a suitable preparation is available. ${ }^{10}$ Reports of the use of both intramuscular and nasogastric clozapine show that this 'assertive' approach can often result in improvements in mental state and a reduction in incidents and segregation, as well as facilitating progression to a less-restrictive environment. Also in practice, a stated intention to use 'enforced' nasogastric or intramuscular clozapine is often sufficient to persuade patients to accept the less intrusive oral route. ${ }^{11,12}$ Clozapine remains an underused treatment and 'enforced' clozapine in particular has been seen as controversial in the UK. ${ }^{13-15}$ The use of, and attitudes to, intramuscular clozapine in the UK have, to date, been described in poster presentations only. ${ }^{16-18}$ We now present the use of intramuscular clozapine in five UK settings with eleven in-patients.

\begin{abstract}
Method
Data was collected between January 2017 and July 2018 at five UK sites: two medium-secure units, two high-secure hospitals and a locked rehabilitation unit. Clinical records were used to identify patients prescribed intramuscular clozapine, and their demographics, previous, response to clozapine use, use of oral and intramuscular therapy, subsequent response as assessed by clinical team impressions, adverse effects from intramuscular treatment and subsequent stabilisation on oral therapy were recorded. Pharmacy staff were consulted to report on the nurses' practical experience of using the intramuscular formulation.
\end{abstract}

\section{How to use intramuscular clozapine}

Clozapine preparation and availability

Clozapine for injection is an unlicensed 'special' product made in the Netherlands by Brocacef and imported to the UK by Durbin PLC via Mawdsleys. The minimum order quantity is two packs of ten $125 \mathrm{mg} / 5 \mathrm{~mL}$ ampoules costing approximately $£ 2000$ in total. Hospital pharmacy departments have experienced delays with importation, supply shortages and stock being sent with a shelf life of only 
2 months. When new, the ampoules have a 2-year shelf life in the dark at $25^{\circ} \mathrm{C}$

\section{Legal authority}

In England and Wales, incapacitous or non-consenting patients detained under the Mental Health Act (1983) may be administered drug treatments for mental disorders for longer than 3 months only if a second opinion appointed doctor approves the treatment, including the route of administration. A rationale needs to be given for the use of unlicensed preparations and so, even if the current authority includes oral and/or intramuscular antipsychotics, a separate request for this preparation will be required. The legal authority to allow enforced blood taking as part of clozapine treatment is provided by section 63 of the Mental Health Act (1983) as long as there is valid authority for the clozapine treatment itself. ${ }^{19}$

\section{Administration and dosing}

Administration is by deep intramuscular injection. Depending on the volume of injection, the gluteal, lateral thigh or deltoid injections sites were used in this series. The Brocacef preparation has no UK licence. The Netherlands licence specifies that volumes of 2-4 mL should be administered into the gluteal muscle and not the arm or thigh, and that volumes $>4 \mathrm{~mL}$ should be given as separate injections. There is no evidence base to favour one site over another. Teams may choose to split doses exceeding $4 \mathrm{~mL}^{20}$ As the bioavailability is approximately twice that of oral preparations, oral doses are halved and injections are usually given once daily. Given the practical limits of administering large volumes of intramuscular medication initial plans for titrations up to volumes of $3.5 \mathrm{~mL}(87.5 \mathrm{mg}$ intramuscular clozapine is equal to $175 \mathrm{mg}$ oral clozapine) may require flexibility, depending on the response. This would enable the use of intramuscular clozapine beyond the initial 14-day titration.

\section{Liaison with clozapine monitoring services}

Although the intramuscular preparation is an unlicensed product, the aim is to establish the patient on oral clozapine as quickly as possible, with as little intramuscular use as possible (preferably none). The treating psychiatrist, pharmacy and patient will need to be registered with a clozapine monitoring service so as to allow oral clozapine to be dispensed. In practice, patients have been registered with a monitoring service. Oral clozapine has been prescribed and, if refused, then intramuscular injections of clozapine administered. Blood monitoring at the required intervals continues, so ensuring that the patient remains registered and that oral clozapine can be dispensed. The clozapine patient-monitoring service manufacturing the relevant oral brand of clozapine has no responsibility for the use of intramuscular clozapine. Our series involved patents registered with all current UK clozapine providers.

\section{Available protocols and guidelines}

Several trusts have produced guidelines and suggested dosing schedules for the use of intramuscular clozapine, which are available online. ${ }^{21}$

\section{Results}

\section{Patient characteristics}

All eleven patients identified were male: ten had a primary diagnosis of schizophrenia (ICD-10 code F20) ${ }^{22}$ and one had a primary diagnosis of bipolar disorder (ICD-10 code F31). ${ }^{22}$ The indication for clozapine was treatment resistance following previous failed treatments, including high dose and antipsychotic polypharmacy. Most had demonstrated a response to clozapine treatment previously, but had discontinued owing to various patient or clinician variables: complaints about blood monitoring, sedation, and a coincidental fall in platelet count owing to immune thrombocytopenia. At least two patients had experienced severe rebound psychosis when oral clozapine was stopped. All the units included have a smoke-free policy, which had been instigated before the data collection period, and so all patients were non-smokers. See Table 1 for a summary of the patient demographics.

\section{Use of intramuscular clozapine}

In three patients the offer to choose between the oral and intramuscular route was sufficient to establish oral clozapine maintenance treatment at between 400 and $425 \mathrm{mg} /$ day, with significant benefit. In the remaining eight patients intramuscular clozapine was required, and between 1 and 99 doses were administered per patient, predominantly into the gluteal muscle, with one being given into the lateral thigh after a patient developed nodules in the gluteal muscle, and one into the deltoid muscle when the patient refused to have the clozapine by any other route (it was the first dose at only $0.25 \mathrm{~mL}$, and no additional effects were noted). Seven patients resisted intramuscular administration to the extent that restraint was used on between one and nine occasions during the initial 14-day dose titration. Restraint was required to take a blood sample in two individuals, one on five occasions and the other on four occasions, and there were no adverse effects during restraint. The remaining patients did not resist. By the end of 5 months, nine patients had been established on oral clozapine, the majority of these showing improvement at doses between $150 \mathrm{mg}$ and $400 \mathrm{mg} /$ day (mean $228 \mathrm{mg} /$ day). No serious adverse effects occurred owing to either the injection itself or associated episodes of restraint. Minor injection site pain occurred in three patients; one experienced sedation, and the patient who had 99 doses of intramuscular clozapine experienced some injection site nodules. There were no injection site abscesses or infections. The maximum reported dose of intramuscular clozapine administered was $250 \mathrm{mg}$ in $10 \mathrm{~mL}$, which was given across three injection sites. Tables 2 and 3 describe the use of intramuscular clozapine in this series.

Serum levels were obtained from two patients who had intramuscular clozapine continuously for five or more days; see Table 4 . The levels were consistent with the equivalent oral doses. ${ }^{23}$ With regards to target plasma levels, all units aimed for the usual recommended plasma range, $0.35-0.6 \mathrm{mg} / \mathrm{L}$, but then would be guided by individual patient symptoms and side-effects; higher than usual levels were used in certain cases. 


\begin{tabular}{|c|c|c|c|c|c|c|}
\hline Patient & $\begin{array}{c}\text { Age at first episode of } \\
\text { psychosis (years) }\end{array}$ & $\begin{array}{c}\text { Age at this admission } \\
\text { (years) }\end{array}$ & $\begin{array}{c}\text { Duration of psychosis at } \\
\text { intramuscular clozapine } \\
\text { prescription (years) }\end{array}$ & $\begin{array}{l}\text { Previous clozapine } \\
\text { response }\end{array}$ & $\begin{array}{l}\text { Previous clozapine } \\
\text { dose }(\mathrm{mg} / \mathrm{d})\end{array}$ & Setting \\
\hline 1 & 17 & 24 & 7 & Poor & 1100 & MSU \\
\hline 2 & 28 & 34 & 6 & Partial & 350 & MSU \\
\hline 3 & 22 & 39 & 17 & Partial & 325 & HSS \\
\hline 4 & 23 & 37 & 14 & $\mathrm{~N} / \mathrm{A}$ & $\mathrm{N} / \mathrm{A}$ & HSS \\
\hline 5 & 21 & 47 & 26 & $\mathrm{~N} / \mathrm{A}$ & $\mathrm{N} / \mathrm{A}$ & MSU \\
\hline 6 & 17 & 37 & 20 & Yes & 250 & MSU \\
\hline 7 & 18 & 30 & 12 & Yes & 600 & MSU \\
\hline 8 & 22 & 36 & 14 & Partial & 200 & HSS \\
\hline$\underline{9}$ & 20 & 31 & 11 & Yes & Not known & HSS \\
\hline 10 & 13 & 50 & 37 & Yes & Not known & HSS \\
\hline 11 & 18 & 38 & 20 & $\mathrm{~N} / \mathrm{A}$ & $\mathrm{N} / \mathrm{A}$ & Rehab \\
\hline Mean & 20 & 37 & 17 & & 471 & \\
\hline
\end{tabular}

MSU, medium-secure unit; HSS, high-secure services; N/A, not applicable; Rehab, low-secure unit.

\section{Prescribing and administering experiences}

Nurses were familiar with the practice of intramuscular antipsychotics and in general terms the procedure was perceived as acceptable: the injection solution was reportedly easy to draw up and, despite the bright yellow colour of the solution, the syringe markings were clearly readable and administration was easy, with very little resistance against the plunger when administering.

Nurses needed reassurance and reminding about maximum volume for single intramuscular administration, so when, for example, $10 \mathrm{~mL}$ was administered, three injections $(4 \mathrm{~mL}+3 \mathrm{~mL}+3 \mathrm{~mL})$ were used. Overall, nurses reported that they were confident in their ability to administer intramuscular clozapine.
Doctors required advice on bioequivalence of oral and intramuscular clozapine and how to word the prescription so that it was clear the intramuscular was only to be used if oral was declined. They also occasionally needed to be reminded to document the rationale for intramuscular treatment in clinical notes and in the associated care plan. Assistance was sometimes needed, especially initially, to complete the required application and gain approval by trust approval bodies.

In the unit where nasogastric clozapine had also previously been used, the procedure for administering intramuscular was seen as much simpler, faster and less stressful for patients. However, the limitations on dosing with the intramuscular formulation owing to volume considerations were a perceived disadvantage in patients who had experienced

\begin{tabular}{|c|c|c|c|c|}
\hline Patient & Route of first clozapine dose & Oral clozapine doses in titration & Intramuscular clozapine doses in titration & Restraint \\
\hline 1 & Oral & 9 & 5 & 5 \\
\hline 2 & Oral & 0 & 14 & 4 \\
\hline 3 & Intramuscular & 10 & 4 & 2 \\
\hline 4 & Oral & 13 & 1 & 7 \\
\hline 5 & Oral & 13 & 1 & 1 \\
\hline 6 & Oral & 14 & 0 & 0 \\
\hline 7 & Oral & 14 & 0 & 0 \\
\hline 8 & Oral & 14 & 0 & 6 \\
\hline 9 & Oral & 14 & 0 & 0 \\
\hline 10 & Intramuscular & 0 & 14 & 2 \\
\hline 11 & Intramuscular & 0 & 14 & 0 \\
\hline Mean & & 9.2 & 5.8 & 2 \\
\hline Total & & 101 & 53 & 22 \\
\hline
\end{tabular}




\begin{tabular}{|lcc|}
\hline Table 3 & $\begin{array}{l}\text { Use of intramuscular clozapine after 14-day initial } \\
\text { titration }\end{array}$ & Intramuscular clozapine doses \\
\hline Patient & 0 \\
\hline 1 & 14 \\
\hline 2 & 5 \\
\hline 3 & 10 \\
\hline 4 & 0 \\
\hline 5 & 0 \\
\hline 6 & 0 \\
\hline 7 & 21 \\
\hline 8 & 0 \\
\hline 9 & 85 \\
\hline 10 & 0 \\
\hline 11 & 135 \\
\hline total & \\
\hline
\end{tabular}

benefit and were poorly compliant on higher doses after the initial titration.

\section{Discussion}

When presented with a patient with TRS who refuses clozapine and consequently faces distress, disability, risk and a potentially extended length of stay, clinicians can feel that they are dealing with an impossible problem. ${ }^{5}$ Alternatives to clozapine are unlikely to work and may well cause harm, although a cycle of depot changes, high dose or polypharmacy regimes may still be attempted. ${ }^{24,25}$ 'Enforced' clozapine (i.e. via nasogastric or intramuscular) is a rarity and, but for the handful of case series above, barely described in the literature; there is believed to be no previous published experience of its use in the UK. Although some NHS trusts have published guidelines for the use of intramuscular clozapine, these do not provide for all eventualities and will likely need adapting to local and individual patient circumstances, often while treatment is taking place; indeed, a flexible and pragmatic approach is more likely to succeed. Difficult decisions will include not only deciding whether to use the intramuscular route at the outset, but also when to stop treatment. Neither oral clozapine nor intramuscular clozapine will provide a solution to every patient with TRS; for example, the intramuscular route may result in patient benefit, but not within the time frame or ceiling dose allowed within a protocol agreed by the governance structure of the institution. In these cases the clinical team may need to consider higher doses and/or multiple

\begin{tabular}{|c|c|c|c|}
\hline $\begin{array}{l}\text { Intramuscular } \\
\text { dose, mg }\end{array}$ & $\begin{array}{c}\text { Equivalent } \\
\text { oral dose, mg }\end{array}$ & $\begin{array}{c}\text { Clozapine, } \\
\text { mg/L }\end{array}$ & $\begin{array}{c}\text { Norclozapine, } \\
\mathrm{mg} / \mathrm{L}\end{array}$ \\
\hline 150 & 300 & 0.26 & 0.11 \\
\hline 125 & 250 & 0.3 & 0.13 \\
\hline
\end{tabular}

intramuscular clozapine injections over the course of the day. A higher-strength solution for injection would be very useful and could enable intramuscular clozapine to be given as the doses increase during the titration, and to continue at higher maintenance doses if the oral dose is refused later in the treatment. Presently one unit in the study prescribes intramuscular clozapine doses lower than the equivalent oral dose if $48 \mathrm{~h}$ of non-adherence is approaching, essentially to prevent the need for retitration. An alternative would include the nasogastric route; however, nasogastric administration of clozapine is culturally less acceptable and less routine than intramuscular in mental health settings. With nasogastric administration, more time is needed in the restraint position, therefore reducing patient safety, and there is a risk the patient could vomit up the medication. The clinical team needs to consider whether the benefits of improvement, possibly in the long term, justify the short-term risks of daily restraint or the complications of using an unlicensed, relatively unused intramuscular drug treatment. Although in our series there were no adverse outcomes from the episodes of restraint, it does not necessarily follow that the procedure is devoid of risk. Our series is unusual compared to the experience elsewhere in that greater number of patients had a much higher number of injections. In the Israeli and Dutch series most patients were established on oral clozapine after one intramuscular dose only, almost all within 2 weeks of starting treatment with clozapine, and only one had treatment for 3 months. It is possible that our sites, which were all secure units, selected for a patient cohort that was more clinically complex and hence more difficult to treat.

In conclusion, given the lack of new treatments for TRS in recent years, it is essential that clinicians deliver the treatments that are likely to work, and clozapine in particular. The intramuscular route can be used to remove one of the impediments to its use, namely refusal of oral treatment. Although our experience was in secure settings, the use of intramuscular antipsychotic medication is used throughout different mental health settings and there is no reason to suggest that 'enforced' clozapine, whether using the intramuscular or any other route, should be confined to secure services. As was found in the series from the Netherlands and with nasogastric clozapine in the UK, many patients accept oral clozapine when faced with the coercive alternative, which is ultimately the desired outcome. There has been concern that the procedure itself might be aversive and painful; however, reports of injection site pain and inflammation were very low. We have demonstrated that the use of intramuscular clozapine, although not without some drawbacks, is overall easy, safe and effective.

\section{About the authors}

Rebecca Henry is the lead pharmacist for education with Southern Health NHS Foundation Trust, UK. Ruth Massey is the lead clinical trials pharmacist and a highly specialist clinical pharmacist with Mersey Care NHS Foundation Trust, UK. Kathy Morgan is a senior clinical pharmacist with Pennine Care NHS Foundation Trust, UK. Johanne Deeks is deputy chief pharmacist in the Secure Care Pharmacy Service at Birmingham and Solihull Mental Health NHS Foundation Trust, UK. Hannah Macfarlane is a clinical tutor 
in psychiatric pharmacy in the School of Life and Health Sciences at Aston University, and the Lead Clinical Pharmacist in Secure Care at Birmingham and Solihull Mental Health NHS Foundation Trust, UK. Nikki Holmes is Head of Pharmacy for Forensic Services in the Pharmacy Department at Nottinghamshire Healthcare NHS Foundation Trust, UK. Edward Silva is a consultant forensic psychiatrist in Secure Services with Mersey Care NHS Foundation Trust, UK.

\section{Acknowledgements}

We thank Peter Phiri R\&D Moorgreen Hospital, Southern Health NHS Foundation Trust, for his advice on the need for ethics approval.

\section{Author contributions}

All authors made contributions to the conception and design of the work, revisions for important intellectual content, approved the final version for publication and are accountable for all aspects of the work.

\section{References}

1 Chakos M, Lieberman J, Hoffman E, Bradford D, Sheitman B. Effectiveness of second-generation antipsychotics in patients with treatment-resistant schizophrenia: a review and meta-analysis of randomized trials. Am J Psychiatry 2001; 158(4): 518-26.

2 Siskind D, McCartney L, Goldschlager R, Kisely S. Clozapine v. first- and second-generation antipsychotics in treatment-refractory schizophrenia: systematic review and meta-analysis. Br J Psychiatry 2016; 209 (5): 385-92.

3 Tiihonen J, Lönnqvist J, Wahlbeck K, Klaukka T, Niskanen L, Tanskanen A, et al. 11-year follow-up of mortality in patients with schizophrenia: a population-based cohort study (FIN11 study). Lancet 2009; 374(9690): 620-7.

4 Frogley C, Taylor D, Dickens G, Picchioni M. A systematic review of the evidence of clozapine's anti-aggressive effects. Int J Neuropsychopharmacol 2012; 15(9): 1351-71.

5 Swinton $M$, Haddock A. Clozapine in special hospital: a retrospective case-control study. J Forensic Psychiatry 2000; 11(3): 587-96.

6 Lokshin P, Lerner V, Miodownik C, Dobrusin M, Belmaker RH. Parenteral clozapine: five years of experience. J Clin Psychopharmacol 1999; 19(5): 479-80.

7 Schulte PF, Stienen JJ, Bogers J, Cohen D, van Dijk D, Lionarons WH, et al. Compulsory treatment with clozapine: a retrospective long-term cohort study. Int J Law Psychiatry 2007; 30(6): 539-45.

8 Kasinathan J, Mastroianni T. Evaluating the use of enforced clozapine in an Australian forensic psychiatric setting: two cases. BMC Psychiatry 2007; 7(Suppl 1): P13.

9 McLean G, Juckes L. Parenteral clozapine (Clozaril). Australas Psychiatry 2001; 9(4): 371.

10 Silva E, Till A, Adshead G. Ethical dilemmas in psychiatry: when teams disagree. BJPsych Adv 2017; 23(4): 231-9.
11 Till A, Selwood J, Silva E. The assertive approach to clozapine: nasogastric administration. BJPsych Bull 2019; 43(1): 21-6.

12 Fisher WA. Elements of successful restraint and seclusion reduction programs and their application in a large, urban, state psychiatric hospital. J Psychiatr Pract 2003; 9(1): 7-15.

13 Pereira S, Beer D, Paton C. Enforcing treatment with clozapine: survey of views and practice. Psychiatrist 1999; 23(6): 342-5.

14 Fahy TJ. Martial arts for psychiatrists. Psychiatrist 2000; 24(5): 197-8.

15 Executive Summary Healthcare Quality Improvement Partnership (HQIP) and The Royal College of Psychiatrists. Report of the Second Round of the National Audit of Schizophrenia (NAS2). Royal College of Psychiatrists, 2014 (https://www.rcpsych.ac.uk/docs/default-source/ improving-care/ccqi/national-clinical-audits/ncap-library/nationalaudit-of-schizophrenia-document-library/nas_round-2-report.pdf? sfvrsn=6356a4b0_2).

16 Henry R, Massey R, Silva E, Qurashi I. An evaluation of intramuscular clozapine in two forensic services. College of Mental Health Pharmacy Annual Conference, Manchester, 2017.

17 Patel S, Wakelam J, Davoren M. A staff attitude survey into the use of intramuscular and nasogastric clozapine in Broadmoor High Secure Hospital. RCPsych Faculty of Forensic Psychiatry Annual Conference, Vienna, 2019

18 Holmes N. Evaluation of intramuscular clozapine. College of Mental Health Pharmacy Annual Conference, Manchester, 2017.

19 Department of Health. Mental Health Act 1983: Code of Practice. TSO, 2015

20 Mason AS, Granacher RP. Clinical Handbook of Antipsychotic Drug Therapy. Brunner/Mazel, 1980.

21 Ritchie G. Clozapine Intramuscular (IM) Guideline, Inpatients Only (18-60 Years of Age Only) Version: 4. Southern Health NHS Foundation Trust, 2019 (http://clinicalskillshub.southernhealth.nhs.uk/EasySiteWeb/ GatewayLink.aspx?alld=109029).

22 World Health Organization, International Classification of Diseases, 10th edition (ICD-10). WHO, 1992.

23 Rostami-Hodjegan A, Amin AM, Spencer EP, Lennard MS, Tucker GT, Flanagan RJ. Influence of dose, cigarette smoking, age, sex, and metabolic activity on plasma clozapine concentrations: a predictive model and nomograms to aid clozapine dose adjustment and to assess compliance in individual patients. J Clin Psychopharmacol 2004; 24(1): 70-8.

24 Correll CU, Rubio JM, Inczedy-Farkas G, Birnbaum ML, Kane JM Leucht S. Efficacy of 42 pharmacologic cotreatment strategies added to antipsychotic monotherapy in schizophrenia: systematic overview and quality appraisal of the meta-analytic evidence. JAMA Psychiatry 2017; 74(7): 675-84

25 Royal College of Psychiatrists. Consensus Statement on High-Dose Antipsychotic Medication. College Report CR190. Royal College of Psychiatrists, 2014 (https://www.rcpsych.ac.uk/docs/default-source/ improving-care/better-mh-policy/college-reports/college-report-cr190. pdf?sfvrsn=54f5d9a2_2). 